\title{
Asymptotic properties of welfare relations
}

\author{
Henrik Petri ${ }^{1}$
}

Received: 17 July 2017 / Accepted: 10 January 2018 / Published online: 22 January 2018

(C) The Author(s) 2018. This article is an open access publication

\begin{abstract}
We introduce and discuss notions of efficiency in the aggregation of infinite utility streams. For any utility streams $x$ and $y$, our efficiency criteria roughly require this: If a utility stream $x$ dominates another utility stream $y$ and if the asymptotic density of the set of coordinates in favor of $x$ is strictly positive, then $x$ is socially preferred to $y$. As a robustness check of the proposed efficiency axioms we explore the consistency of the axioms with notions of equity. Our main results characterize one period utility domains, i.e., the set of utilities $Y$ attainable by each generation, admitting a social welfare aggregator with the desired properties.
\end{abstract}

Keywords Social welfare $\cdot$ Asymptotic density $\cdot$ Utility aggregation

JEL Classification C65 - D60 - D70 - D90

\section{Introduction}

We propose and discuss criteria of efficiency in the aggregation of infinite utility streams. A common approach is to require efficiency in the Pareto sense. However, as noted by several authors (Koopmans 1960; Diamond 1965; Basu and Mitra 2003; Fleurbaey and Michel 2003; Banerjee 2006; Zame 2007; Hara et al. 2008; Crespo et al.

This paper is based on a chapter of my Ph.D. Dissertation at Stockholm School of Economics. I thank Mark Voorneveld, Tommy Andersson and two anonymous referees for helpful comments. Financial support by the Wallander-Hedelius Foundation under Grant P2014-0189:1 is gratefully acknowledged.

$凶$ Henrik Petri

henrik@petri.se

1 Stockholm Business School, Stockholm University, Företagsekonomiska institutionen, 10691 Stockholm, Sweden 
2009; Dubey and Mitra 2011, 2015), a fundamental problem with this approach is that the Pareto axiom is generally inconsistent with equity as formalized by anonymity. ${ }^{1}$ This will be discussed in more detail later in the introduction. To circumvent the problem we propose weaker versions of some well-known Pareto axioms. For any utility streams $x$ and $y$, our criteria roughly speaking require this: If a utility stream $x$ dominates another utility stream $y$ and if the (lower asymptotic) density of the set of coordinates in favor of $x$ is strictly positive, then $x$ is socially preferred to $y$.

A crucial ingredient in our formalization of efficiency is the notion of asymptotic density (or natural density), which is a notion designed to measure how large certain subsets of the natural numbers are. It formalizes the intuition that the set of even numbers is larger than the set of multiples of three, even though there is a one to one correspondence between them (both are countable). Heuristically, if we let the set of natural numbers have measure one, the even numbers should have measure $\frac{1}{2}$, since "half" of the members of $\mathbf{N}$ are even, and similarly the set of multiples of three should be assigned measure $\frac{1}{3}$.

Part of our motivation is that existing notions of efficiency, as formalized by various Pareto principles, are economically either too strict or too weak. Crespo et al. (2009) argue that the strong Pareto axiom is too strict since the welfare of a society should not be influenced by the utility of a single generation. In consequence they introduce the infinite Pareto principle. ${ }^{2}$ But even infinite Pareto seems too strict economically since, intuitively, the welfare of a society should not be affected by the utilities of certain "small" subsets of generations. To see this, suppose that any generation $n$ with $n=10^{k}$ receive an increase in their utility. According to infinite Pareto the new stream of utilities should be ranked above the old stream. However, the distance between any consecutive pair of generations receiving a strict increase in utility goes to infinity as $k$ increases. Hence, it is not clear that the new stream of utilities should be ranked strictly above the old stream.

The weak Pareto axiom takes the issues mentioned in the previous paragraph into account and demands that if every generation is strictly better off then society should be strictly better off. Hence, it is insensitive to an increase in the utilities of a "small" subset of generations. However, this axiom seems too weak economically. Suppose for example that every second generation, $n=2 k$ for $k \in \mathbf{N}$, receives an increase in their utility and every other generations' utility remains the same. Then, it seems intuitively clear that the former stream should be ranked above the latter; however, the weak Pareto axiom does not necessarily see the difference.

As a suggested remedy to the issues mentioned above, we propose a criterion that we call asymptotic Pareto. It is intermediate in strength between infinite Pareto and weak Pareto. Roughly speaking, it requires the following: If every generation receives a higher utility, and if every generation in a set of strictly positive lower asymptotic density receives a strict increase in utility, then society is better off.

\footnotetext{
${ }^{1}$ Anonymity requires the social welfare of a stream of utilities to be unchanged by permuting the welfare of finitely many generations.

${ }^{2}$ Infinite Pareto requires a stream of utilities to be ranked above another stream if every generation receive a higher utility compared to the other stream, and an infinite number of generations receive a strictly higher utility.
} 
In addition, we propose a weak version of asymptotic Pareto. Consider utility streams $x=(1,1,1,1,1,1,1, \ldots)$ and $y=(1,0.99,1,0.999,1,0.9999,1, \ldots)$. According asymptotic Pareto $x$ is socially preferred to $y$. But since, for any given threshold, there are at most a finite number of generations receiving an increase above the threshold it is not clear that $x$ should be ranked above $y$. Weak asymptotic Pareto takes this into account by ranking a stream of utilities above another stream only if a subset of generations with positive lower asymptotic density receive an increase exceeding some fixed threshold $\varepsilon>0$.

Due to its intuitive interpretation, asymptotic density has gained considerable interest as a tool in the theory of social welfare aggregation. Notable are the papers by Lauwers (1998), Lauwers (2011) and Fey (2004, 2005). Fey (2004) characterizes majority rules with infinitely many voters, and uses asymptotic density to measure the size of large societies. In his characterization, Fey (2004) uses a criterion called density positive responsiveness, which requires ties to be broken if a set of voters with positive asymptotic density change their preference. The criterion that we propose is also sensitive to societies of positive asymptotic density. Lauwers (1998) considers an equity principle based on asymptotic density, bounded anonymity. He restricts attention to linear social welfare functions and discusses the compatibility of bounded anonymity with efficiency. One implication of his work is that, although strong Pareto is generally incompatible with bounded anonymity, a weak form of the Pareto axiom is not. We discuss the relation between our and his results further below.

The conflict between equity and efficiency is well known in the literature on intertemporal welfare economics (Koopmans 1960; Diamond 1965; Basu and Mitra 2003). It has been noted that equity, as formalized by anonymity, generally is inconsistent with the Pareto principle. Basu and Mitra (2003) show that there is no social welfare function with these properties. Even if one restricts attention to social welfare orders, anonymity and Pareto, implies the existence of a non-Ramsey set, and is thus a non-constructive object (Lauwers 2010). The literature has found several ways around the problems posed above. For instance, one could agree to drop the completeness axiom and several interesting relations emerge satisfying Pareto and anonymity. See for instance (Basu and Mitra 2007; Bossert et al. 2007; Zuber and Asheim 2012) and references therein. Alternatively one could consider other versions of equity and efficiency as in Basu and Mitra (2007), Dubey and Mitra (2011, 2015) and Alcantud (2012, 2013). Our proposed solution is to consider weakened versions of the Pareto axiom.

As a robustness check of our efficiency axioms and as a main goal of the paper, we investigate the "degree of conflict" between the proposed efficiency principles and various notions of equity. The paper is divided into two parts.

In the first part of the paper, we characterize one period utility domains $Y$ admitting a social welfare function satisfying asymptotic Pareto and finite anonymity. Thus, we identify the exact limitations imposed by our axioms on the domain of a prospective social welfare function. The results are analogous to the results obtained in Dubey and Mitra (2011) and Dubey and Mitra (2015).

In the second part of the paper, we then proceed to derive limitations imposed on $Y$ by a social binary relation satisfying asymptotic Pareto and bounded anonymity. We 
do not impose any explicit transitivity/completeness requirements on welfare relations considered in this part of the paper.

To see why it may be of economic interest to consider bounded anonymity, in addition to finite anonymity, note that finite anonymity only requires every single generation to count the same in social evaluation. However, it does not necessarily require sets of generations with "equal size" to count the same. Consider for example utility streams $x=(1,0,1,0,1, \ldots)$ and $y=(0,1,0,1,0, \ldots)$. A strict preference between $x$ and $y$ is consistent with finite anonymity. But intuitively it could be argued that these two sequences should be regarded as equally important, since in both cases "half of the generations" receive a utility of one. Bounded anonymity demands a social welfare order to be left invariant by permutations switching subsets of equally large lower asymptotic density. Thus bounded anonymity implies that society regards $x$ and $y$ as equally important.

Since every function is a binary relation, one would expect the conditions on $Y$ derived in the second part of the paper to be more permissive than the corresponding conditions obtained in the first part of the paper. The answer to this question depends on whether we impose finite or bounded anonymity as an axiom on welfare aggregators. By theorem 2 in Svensson (1980), there is a social welfare relation satisfying finite anonymity and (asymptotic) Pareto on arbitrary domains $Y \subseteq[0,1]$. However, by assuming bounded anonymity, it turns out that the domains $Y$ admitting a binary relation satisfying bounded anonymity and asymptotic Pareto exactly coincides with domains $Y$ admitting a social welfare function satisfying finite anonymity and asymptotic Pareto.

A corollary to the results discussed above is that there is a social welfare function satisfying bounded anonymity and asymptotic Pareto if and only if there is a binary relation with these properties. This result is in quite sharp contrast to previous results in the literature. For example, Dubey and Mitra (2011) derive characterizing conditions on $Y$ such that $Y$ admits a social welfare function satisfying weak Pareto and finite anonymity. However, for all subsets $Y$ that fail to admit such a function there is a social welfare relation with the desired properties (theorem 2 in Svensson 1980).

The paper is organized as follows. Preliminaries are in Sect. 2. The model and efficiency axioms are introduced and discussed in Sect. 3. Section 4, the first part of the paper, is devoted to the discussion of the consistency between asymptotic Pareto and anonymity. The second part of the paper, Sect. 5 then discusses the consistency of bounded anonymity with our proposed efficiency principles. We conclude in Sect. 6.

\section{Notation and preliminaries}

Notation: A binary relation $\succsim$ on a set $X$ is a subset $\succsim \subseteq X \times X$. As usual, we denote by $\sim$ the symmetric part of $\succsim$ and by $\succ$ the asymmetric part of $\succsim$. Bold letters $\mathbf{N}, \mathbf{Z}$ and $\mathbf{R}$ are used to denote the set of natural numbers, integers and real numbers. It is assumed that $0 \notin \mathbf{N}$. For every $n \in \mathbf{N}$ we set $[1, n]:=\{1, \ldots, n\}$.

A set $X$ with order $\succsim_{X}$ is order isomorphic to a set $Y$ with order $\succsim_{Y}$ if and only if there is a bijective function $f: X \rightarrow Y$ such that $x \succsim_{X} y$ if and only if $f(x) \succsim_{Y} f(y)$. We will denote by $w$ the natural numbers with canonical order $\geq$ and by $w^{*}$ the natural 
numbers with reverse order $\leq$ (defined by $y \leq x$ if and only if $y \geq x$ for all $x, y \in X)$. Alternatively, $w^{*}$ can be defined as the set of negative integers $\mathbf{M}$ with order inherited from $\mathbf{Z}$.

Asymptotic density: We recall some basic notions from the theory of uniformly distributed sequences and natural densities. For a thorough account see Kuipers and Niederreiter (2012). As mentioned in the introduction, the asymptotic density of a set is designed to capture our intuition that the set of even numbers has "half the size" of the set of natural numbers. Let $|A|$ denote the cardinality of a finite set $A$. The lower (upper) asymptotic density of a set of natural numbers is defined as:

$$
\underline{d}(A):=\liminf _{n \rightarrow \infty} \frac{|A \cap[1, n]|}{n} \quad\left(\bar{d}(A):=\limsup _{n \rightarrow \infty} \frac{|A \cap[1, n]|}{n}\right)
$$

for all $A \subseteq \mathbf{N}$. If $\underline{d}(A)=\bar{d}(A)$, we denote their common value by $d(A)$, which is the asymptotic density of $A$. The collection of sets with an asymptotic density is denoted $\mathcal{A}$. For a large class of sets, the asymptotic density is not defined, the collection $\mathcal{A}$ is not a Boolean algebra and $d$ is not a finitely additive measure. However, by use of the Hahn Banach theorem, it is possible to extend $d$ to a finitely additive density measure $e^{3}$ defined on the algebra of all subsets of natural numbers. Another approach is to use free ultrafilters as outlined in Fey (2005) and Lauwers (1998). Also note that the lower (upper) asymptotic density is defined for all subsets of natural numbers.

A sequence $\left(x_{n}\right)$ in $\mathbf{R}$ is said to be uniformly distributed modulo 1 if

$$
\lim _{n \rightarrow \infty} \frac{\left|\left\{k \in \mathbf{N}: x_{k} \in[a, b)\right\} \cap[1, n]\right|}{n}=b-a
$$

for all $a, b \in[0,1]$. An example of a uniformly distributed sequence, is the binary Van der Corput sequence, defined as follows: For every $n \geq 2$ let $n-1=\sum_{i=0}^{k} a_{i} 2^{i}$ be the binary expansion of $n-1$ and set $x_{n}:=\sum_{i=0}^{k} \frac{a_{i}}{2^{i+1}}$ and $x_{1}:=0$. The binary Van der Corput sequence plays a crucial role in the proof of Lemma 4.5. It is a standard fact in the theory of uniformly distributed sequences that the binary Van der Corput sequence is uniformly distributed modulo $1 .^{4}$

Permutations: A permutation is a bijective function $\pi: \mathbf{N} \rightarrow \mathbf{N}$. A permutation $\pi: \mathbf{N} \rightarrow \mathbf{N}$ is a finite permutation if there is a $n \in \mathbf{N}$, such that $\pi(k)=k$ for all $k \geq n$, i.e., $\pi$ "switches" a finite number of coordinates. A permutation $\pi: \mathbf{N} \rightarrow \mathbf{N}$ is a bounded permutation if

$$
\lim _{n \rightarrow \infty} \frac{|\{k: k \leq n<\pi(k)\}|}{n}=0
$$

\footnotetext{
3 For a statement and proof of the Hahn-Banach theorem see Banach (1987), p. 18. Page 19, section 4 of Banach (1987) contains a construction of Banach Limits from which the existence of a density measure directly follows. A Banach limit is a continuous linear functional extending the ordinary limit operator to the set of all bounded sequences.

4 This fact follows by theorem 1.1 (page 89) together with theorem 3.5 (page 127) in Kuipers and Niederreiter (2012).
} 
Roughly speaking, a bounded permutation switches subsets of equally large asymptotic density. The permutation $\pi: \mathbf{N} \rightarrow \mathbf{N}$ defined by $\pi(1)=1, \pi(n)=n+1$ if $n$ is even and $\pi(n)=n-1$ otherwise (i.e., swapping the odd and even numbers, except $n=1$ ) is a bounded permutation. The set of all bounded permutations is denoted by $G$. Similarly, we denote the set of all finite permutations by $G_{f}$. For every sequence $x=\left(x_{n}\right)$ and permutation $\pi: \mathbf{N} \rightarrow \mathbf{N}$ we let, with a slight abuse of notation, $\pi \circ x$ denote the sequence $\left(x_{\pi(n)}\right)$ obtained after permuting the coordinates of $x$.

\section{Welfare aggregators and axioms}

In this section, we define welfare aggregators on infinite utility streams, and then move on to discuss axioms for such welfare aggregators.

Let $Y \subseteq \mathbf{R}$ be a non-empty set of utilities attainable by each generation. Then $X=Y^{\mathbf{N}}$ is the set of all utility streams. If $x=\left(x_{k}\right) \in X$ then $x=\left(x_{1}, x_{2}, \ldots, x_{n}, \ldots\right)$ and, for all $n \in \mathbf{N}, x_{n} \in Y$ is the utility experienced by generation $n$. A social welfare function (SWF) is a function $W: X \rightarrow \mathbf{R}$, a social welfare order (SWO) is a reflexive, transitive and complete order $\succsim o$ on $X$. Similarly a binary welfare relation (BWR) is a binary relation $\succsim_{R}$ on $X$. A distinguishing feature of a binary welfare relation, compared to any other binary relation, is its domain of definition $X$.

\subsection{Axioms and discussion}

We introduce some axioms for binary welfare relations. Since every social welfare function/order is a binary welfare relation, whenever we say that a social welfare function/order satisfies a certain axiom, we mean that the induced binary welfare relation satisfies it. A list of efficiency (Pareto) axioms, roughly ordered in decreasing order of logical strength, is now given:

P Pareto For all $x, y \in X$, if $x \geq y$ and $x_{i}-y_{i}>0$ for some $i \in \mathbf{N}$, then $x \succ_{R} y$.

IP Infinite Pareto For all $x, y \in X$, if $x \geq y$ and $x_{i}-y_{i}>0$ for all $i \in I$ for some infinite subset $I \subseteq \mathbf{N}$, then $x \succ_{R} y$.

AP Asymptotic Pareto For all $x, y \in X$, if $x \geq y$ and there exists a subset $A$ of $\mathbf{N}$ with $\underline{d}(A)>0$ such that $x_{i}-y_{i}>0$ for all $i \in A$, then $x>_{R} y$.

WAP Weak asymptotic Pareto For all $x, y \in X$, if $x \geq y$ and there exists a subset $A$ of $\mathbf{N}$ with $\underline{d}(A)>0$ and an $\varepsilon>0$ such that $x_{i}-y_{i}>\varepsilon$ for all $i \in A$, then $x \succ_{R} y$.

WP Weak Pareto For all $x, y \in X$, if $x_{i}-y_{i}>0$ for all $i \in \mathbf{N}$, then $x \succ_{R} y$.

Some other axioms that we will use/discuss are listed below.

A Anonymity For all $\pi \in G_{f}, \pi \circ x \sim_{R} x$.

BA Bounded anonymity For all $\pi \in G, \pi \circ x \sim_{R} x$.

We comment on some simple relationships between the axioms. It is clear that $\mathrm{P}$ $\Longrightarrow \mathrm{IP} \Longrightarrow \mathrm{AP} \Longrightarrow$ WAP, and that AP $\Longrightarrow$ WP. However, the relationship between WP, AP and WAP is a little subtle. Consider for example utility streams 
$x=(1 / n)_{n=1}^{\infty}$ and $\mathbf{0}$ (where $\mathbf{0}$ is the utility stream with all one period utilities equal to zero). A social welfare function satisfying WP or AP ranks $x$ above $\mathbf{0}$. But an SWF satisfying WAP is not necessarily able to rank $x$ above $\mathbf{0}$ : for every $\varepsilon>0$ there are at most finitely many $i \in I$ such that $x_{i}>\varepsilon$, and since every finite subset of natural numbers has lower asymptotic density zero, the hypothesis of WAP does not hold. An example of a social welfare function satisfying WAP and $x \sim \mathbf{0}$ is the function in Sect. 4.1, equation (1). None of the implications above can be reversed without adding additional hypotheses. For instance, if $Y$ is finite then a social welfare function $W: X \rightarrow \mathbf{R}$ satisfies WAP if and only if it satisfies AP.

In Basu and Mitra (2003) it was shown that there is no social welfare function satisfying anonymity A and Pareto P. If we agree that an SWF should satisfy the finite anonymity axiom A, then in light of the impossibility results by Basu and Mitra (2003), we should look for a weakening of the Pareto axiom. Several weakenings of the Pareto axiom have been considered in the literature, such as the infinite Pareto axiom (IP) in Crespo et al. (2009). Even though IP, a priori, seems like a considerably weaker condition than $P$, the quite surprising result by Crespo et al. (2009) shows that there is no social welfare function satisfying infinite IP and A. Another very weak version of the Pareto axiom, the weak Pareto axiom, was studied in Dubey and Mitra (2011), where one period utility domains $Y$, admitting an SWF satisfying WP and A, were characterized. Asymptotic Pareto fits in between weak Pareto WP and infinite Pareto IP in logical strength, and hence we expect a characterizing condition on $Y$ to be stronger than the condition in Dubey and Mitra (2011). As we will show finite $Y$ is both necessary and sufficient for the existence of a social welfare function satisfying asymptotic Pareto AP and anonymity A.

Lauwers (1998) mentions an efficiency concept similar to weak approximate Pareto WAP, which we state below.

LWAP Lauwers' weak asymptotic Pareto For all $x, y \in X$, if $x \geq y$ and there exists a subset $A$ of $\mathbf{N}$ with $d(A)>0$ and an $\varepsilon>0$ such that $x_{i}-y_{i}>\varepsilon$ for all $i \in A$, then $x \succ_{R} y$.

Note that LWAP is verbatim identical to WAP, except that the occurrence of lower asymptotic density $\underline{d}$ is replaced with asymptotic density $d$. Our efficiency principle is less permissive than Lauwers' since any social welfare function satisfying WAP satisfies his efficiency axiom. However, WAP imposes a similar restriction on one period utility domains, as Lauwers' criteria does.

The following axiom corresponds to our AP-axiom but replaces lower asymptotic density with asymptotic density.

dAP $d$-Asymptotic Pareto For all $x, y \in X$, if $x \geq y$ and there exists a subset $A$ of $\mathbf{N}$ with $d(A)>0$ such that $x_{i}-y_{i}>0$ for all $i \in A$, then $x \succ_{R} y$.

Axiom dAP is not considered by Lauwers (1998). He deals with welfare aggregators defined on bounded sequences (with ranges $x(\mathbf{N})$ that are possibly infinite.) Adopting the arguments in the present paper one can show that there is no welfare function satisfying $\mathrm{dAP}$ and $\mathrm{A}$ on the set of bounded sequences. This may be why axiom dAP is not discussed by him. 


\section{Part 1: The conflict between finite anonymity and efficiency}

In this section, we characterize one period utility domains $Y$ admitting a social welfare function satisfying (weak) asymptotic Pareto and anonymity. The theorem below summarizes the main results of part 1 of the paper.

Theorem 4.1 Let $X=Y^{\mathbf{N}}$, where $Y \subseteq \mathbf{R}$ is non-empty.

1. There is a social welfare function $W: X \rightarrow \mathbf{R}$ satisfying asymptotic Pareto AP and anonymity $A$ if and only if $Y$ is finite.

2. There is a social welfare function $W: X \rightarrow \mathbf{R}$ satisfying weak asymptotic Pareto WAP and anonymity $A$ if and only if $Y$ is bounded.

The theorem above is convenient in that it helps to identify domains admitting a social welfare aggregator satisfying asymptotic Pareto and anonymity. It also serves as a consistency check of the proposed efficiency axioms, since it is not a priori clear that a social welfare aggregator satisfying these properties exists. Before proving Theorem 4.1, we give some examples illustrating the usefulness of the result.

Example 4.1 Let $Y=\left\{\frac{1}{n}\right\}_{n=1}^{\infty}$. Clearly $Y$ is not finite and hence by Theorem 4.1 it follows that there is no SWF satisfying AP and A on $Y$. However, since $Y$ is a bounded subset of $\mathbf{R}$, by weakening AP to WAP, we see by Theorem 4.1 again, that there is an SWF satisfying A and WAP.

Example 4.2 Let $Y=\mathbf{N}$. Then $Y$ is neither finite nor bounded. Hence, there is no social welfare function satisfying (weak) asymptotic Pareto WAP and anonymity A on $Y$.

Example 4.3 Let $Y=\mathbf{N}$ and let $W: X \rightarrow \mathbf{R}$ be a social welfare function satisfying anonymity. Then $W: X \rightarrow \mathbf{R}$ cannot satisfy either AP or WAP.

We proceed to discuss the proof of Theorem 4.1. The first subsection (section 4.1) proves the "if"-direction of the claim by giving an example of a social welfare function with the stated properties. In the second subsection (Sect. 4.2), we discuss the other direction of Theorem 4.1 by deriving the exact limitations imposed on $Y$ by a social welfare function satisfying A and AP.

\subsection{Positive results}

The main goal of this section is to exhibit an example of a social welfare function $W: Y^{\mathbf{N}} \rightarrow \mathbf{R}$ satisfying anonymity A and asymptotic Pareto AP (WAP) on finite (bounded) domains $Y$.

Let $W_{n}(x):=\frac{1}{n} \sum_{k=1}^{n} x_{k}$ for all $n \geq 1$ and $x \in X$. Our candidate social welfare function, satisfying anonymity A and asymptotic Pareto AP, is the function $W: X \rightarrow$ $\mathbf{R}$ defined by:

$$
W(x):=\liminf _{n \rightarrow \infty} W_{n}(x)=\liminf _{n \rightarrow \infty} \frac{1}{n} \sum_{k=1}^{n} x_{k}
$$


for all $x \in X$. Note that $W: X \rightarrow \mathbf{R}$ is well defined whenever $Y$ is bounded. ${ }^{5}$ It is easy to see that $W(x)$ satisfies finite anonymity A: The limit inferior of a sequence does not depend on the values assumed by a finite number of its terms. In Proposition 4.3, we show the stronger claim that $W$ satisfies bounded anonymity BA. We will discuss the consistency of BA with AP and WAP further in section 5.

In part 1 of Proposition 4.3, we show that $W: X \rightarrow \mathbf{R}$ satisfies WAP and BA whenever $Y \subseteq \mathbf{R}$ is bounded. The existence of a social welfare function satisfying AP and BA on finite $Y$ is then derived as a corollary in part 2 of the proposition.

In our proof that $W: X \rightarrow \mathbf{R}$ satisfies asymptotic Pareto and bounded anonymity we will need Lemma 4.2, showing that the Cesáro mean, $\lim _{n \rightarrow \infty} \frac{1}{n} \sum_{k=1}^{n} x_{k}$, of a sequence $x \in X$, with bounded one period utilities, is invariant under the action of bounded permutations:

Lemma 4.2 If $\pi \in \mathrm{G}$ and $Y \subseteq \mathbf{R}$ is bounded, then

$$
\lim _{n \rightarrow \infty}\left(W_{n}(x)-W_{n}(\pi \circ x)\right)=0
$$

for all sequences $\left(x_{k}\right)$ with $x_{k} \in Y$.

We provide a Proof of Lemma 4.2 in Appendix A.1. We are now ready to give our main result of this section.

Proposition 4.3 Let $X=Y^{\mathbf{N}}$ and $Y \subseteq \mathbf{R}$ a non-empty set. Define $W: X \rightarrow \mathbf{R}$ by $W(x):=\liminf _{n \rightarrow \infty} W_{n}(x)$ for all $x=\left(x_{k}\right) \in X$.

1. If $Y$ is bounded then $W: X \rightarrow \mathbf{R}$ satisfies bounded anonymity $B A$ and weak asymptotic Pareto WAP.

2. If $Y$ is finite then $W: X \rightarrow \mathbf{R}$ satisfies bounded anonymity $B A$ and asymptotic Pareto AP.

Proof (Part 1) Weak asymptotic Pareto: Assume that $x, y \in X$ are such that $x_{i} \geq y_{i}$ for all $i \in \mathbf{N}$ and that there is a subset $A$ of $\mathbf{N}$ with $\underline{d}(A)>0$ and an $\varepsilon>0$ such that $x_{i}-y_{i}>\varepsilon$ for all $i \in A$. Since $x_{i}-y_{i}>\varepsilon>0$ for all $i \in A$ we have:

$$
\begin{aligned}
W(x-y) & =\liminf _{n \rightarrow \infty} W_{n}(x-y)=\liminf _{n \rightarrow \infty} \frac{1}{n} \sum_{i=1}^{n}\left(x_{i}-y_{i}\right) \geq \\
& \geq \liminf _{n \rightarrow \infty} \varepsilon \frac{|A \cap[1, n]|}{n}=\varepsilon \underline{d}(A)>0 .
\end{aligned}
$$

Note that $W(x) \geq W(x-y)+W(y)$ by super additivity ${ }^{6}$ of the liminf-operator. By above it hence follows that $W(x) \geq W(x-y)+W(y) \geq \varepsilon \underline{d}(A)+W(y)>W(y)$. Thus $W: X \rightarrow \mathbf{R}$ satisfies weak asymptotic Pareto WAP.

\footnotetext{
5 Since $Y$ is bounded there is an $M \in \mathbf{R}$ such that $\left|x_{k}\right|<M$ for all $k \in \mathbf{N}$. Hence, for all $n \in \mathbf{N}$ : $\left|W_{n}(x)\right|=\left|\frac{1}{n} \sum_{k=1}^{n} x_{k}\right| \leq \frac{1}{n} \sum_{k=1}^{n}\left|x_{k}\right| \leq \frac{n M}{n}=M$. But this implies that all subsequential limits of the sequence $\left(W_{n}(x)\right)$ belong to $[-M, M]$ and hence in particular $W(x)=\liminf _{n \rightarrow \infty} W_{n}(x) \in[-M, M]$.

${ }^{6}$ By Rudin (1964), [Ex.5,p.78] it follows that $\lim \sup \left(x_{n}+y_{n}\right) \leq \lim \sup x_{n}+\lim \sup y_{n}$. Hence by noting that lim inf $x_{n}=-\lim \sup -x_{n}$ for all sequences $\left(x_{n}\right)$ we have $\lim \inf \left(x_{n}+y_{n}\right)=-\lim \sup -\left(x_{n}+y_{n}\right) \geq$ $-\lim \sup -x_{n}-\lim \sup -y_{n}=\lim \inf x_{n}+\lim \inf y_{n}$.
} 
Bounded anonymity: Let $\pi \in G$, then by Lemma 4.2 it follows that $\lim _{n \rightarrow \infty}\left(W_{n}(x)-\right.$ $\left.W_{n}(\pi \circ x)\right)=0$, but then also $\liminf _{n \rightarrow \infty}\left(W_{n}(x)-W_{n}(\pi \circ x)\right)=0$, and hence $W(x-\pi \circ x)=0$. Similar reasoning gives $W(\pi \circ x-x)=0$. By super additivity of limit inferior we have $W(x) \geq W(x-\pi \circ x)+W(\pi \circ x)=W(\pi \circ x)$ and $W(\pi \circ x) \geq W(\pi \circ x-x)+W(x)=W(x)$. Hence, $W(\pi \circ x)=W(x)$.

(Part 2) This part of the proposition follows immediately by part 1 and by noting that if $Y$ is finite then there is an $\varepsilon>0$ such that $|x-y|>\varepsilon$ for all distinct $x, y \in Y$.

There are many other social welfare functions satisfying A and AP. By use of the Hahn Banach theorem, it is possible to define a welfare function by letting $W(x)=$ $\int x d \mu$, where $\mu: 2^{\mathbf{N}} \rightarrow[0,+\infty]$ is a density measure.

\subsection{Negative results}

The result in Sect. 4.1 implies that there is a social welfare function satisfying AP (WAP) and A on $X=\mathbf{Y}^{\mathbf{N}}$ whenever $Y$ is finite (bounded). The main result of this section, Proposition 4.4, shows that finite (bounded) $Y$ is also necessary for the existence of a social welfare function with the desired properties. This completes the proof of Theorem 4.1 .

Proposition 4.4 Let $X=Y^{\mathbf{N}}$, where $Y \subseteq \mathbf{R}$ is non-empty.

1. If there is a social welfare function $W: X \rightarrow \mathbf{R}$ satisfying asymptotic Pareto AP and anonymity $A$ then $Y$ is finite.

2. If there is a social welfare function on $W: X \rightarrow \mathbf{R}$ satisfying weak asymptotic Pareto WAP and anonymity $A$ then $Y$ is bounded.

To prove Proposition 4.4, part 1, we first note that every infinite subset $Y$ of $\mathbf{R}$ contains a subset order isomorphic to $w$ or $w^{*}$ (see Lemma A.3 in Appendix A.3). The existence of an SWF $W: X \rightarrow \mathbf{R}$ satisfying A and AP on $X$ with infinite $Y$ then implies the existence of an SWF with the same properties on $X=Y^{\mathbf{N}}$ where $Y$ is the set of natural numbers $\mathbf{N}$. The main technical difficulty is then to show Lemma 4.5 implying that there is no SWF satisfying A and AP whenever $Y=\mathbf{N}$.

Part 2 of Proposition 4.4 follows using similar arguments as in part 1. First Lemma A.4 in Appendix A.4 shows that every unbounded subset of $\mathbf{R}$ contains a subset order isomorphic to $w$ or $w^{*}$ without any accumulation points. Using this, the existence of an SWF satisfying WAP and A on an unbounded subset $Y$ of $\mathbf{R}$ implies the existence of an SWF with such properties on $X=Y^{\mathbf{N}}$ where $Y=\mathbf{N}$. Hence, as for part 1, the proof of part 2 reduces to showing Lemma 4.5. We now state Lemma 4.5 and give some comments on the strategy of proof.

Lemma 4.5 Let $Y=\mathbf{N}$ and $X=Y^{\mathbf{N}}$. Then, there is no social welfare function $W: X \rightarrow \mathbf{R}$ satisfying anonymity $A$ and asymptotic Pareto AP.

The full Proof of Lemma 4.5 is in Appendix A.2. The technique is inspired by a similar technique in Dubey and Mitra (2011), which in turn is a (very) refined version of an argument, attributed to Debreu (1954), showing that the lexicographic order on 
$\mathbf{R}^{2}$ is not representable by a utility function. The basic idea of proof is the following. By using asymptotic Pareto and finite anonymity, for every $\alpha \in(0,1)$ we construct sequences $x(\alpha), z(\alpha) \in X$ such that $W(z(\alpha))>W(x(\alpha))$ and $W(x(\beta))>W(z(\alpha))$ whenever $1>\beta>\alpha$.

The sequences constructed above and a standard argument Debreu (1954) then implies an injection from $\mathbf{R}$ to $\mathbf{Q}$, contradicting countability of $\mathbf{Q}$. More precisely the argument goes as follows. For every $t \in(0,1)$ define an interval [ $W(x(t)), W(z(t))]$. Since $W(z(t))>W(x(t))$ the interval is non-empty and by density of the rational numbers we may pick a rational number $q_{t} \in[W(x(t)), W(z(t))]$ for every $t \in(0,1)$. Define a function $f:(0,1) \rightarrow \mathbf{Q}$ by $f(t):=q_{t}$. We claim that $f$ is injective. Let $s>t$ then $W(x(s))>W(z(t))$ and hence $[W(x(t)), W(z(t))]$ and $[W(x(s)), W(z(s))]$ are disjoint, in turn implying that $q_{s} \neq q_{t}$. Thus $f$ is an injection from $(0,1)$ to $\mathbf{Q}$.

We briefly explain how the technique used to prove the impossibility result in Dubey and Mitra (2011) is adapted to our setting. In their proof subsets $A(t)$ of $\mathbf{N}$ corresponding to Dedekind cuts are used, i.e. subsets of the form $A(t)=\{n \in \mathbf{N}$ : $\left.r_{n} \in[0, t)\right\}$ for $t \in \mathbf{R}$ where $\left(r_{n}\right)$ is some enumeration of the rational numbers. These sets have the property that $A(t) \backslash A(s)$ is infinite whenever $s<t .{ }^{7}$ However, for our purposes an even stronger property is needed. We would like to find an uncountable collection of sets $A(t)$ such that $A(t) \backslash A(s)$ has positive asymptotic density whenever $s<t$. The solution is to let $\left(r_{n}\right)$ not just be any enumeration of the rational numbers, but a specific one, the binary Van der Corput sequence $\left(d_{n}\right)$. As explained in section 2 the binary Van der Corput sequence is uniformly distributed modulo 1 (by well-known results), and hence sets of the form $A(t):=\left\{n \in \mathbf{N}: d_{n} \in[0, t)\right\}$ have the desired property. These sets are then used to define sequences $x(\alpha), z(\alpha) \in X$ with properties mentioned above.

By inspecting the Proof of Lemma 4.5 in Appendix A.2, it is evident that we only need to use asymptotic density $d$ (instead of $\underline{d}$ as in the definition of AP) in order to derive a contradiction. Hence, Lemma 4.5 directly implies that no social welfare function satisfying A and LWAP exists for $Y=\mathbf{N}$. It is clear that if an SWF is such that it satisfies WAP then it satisfies LWAP (Since if $d(A)>0$ then $\underline{d}(A)>0$ ). Hence, the SWF constructed in Proposition 4.1 satisfies LWAP. It follows that the characterizing condition on $Y$ such that $Y$ admits an SWF satisfying LWAP and A is identical to the characterizing condition on $Y$ such that $Y$ admits an SWF satisfying WAP and A.

Corollary 4.6 Let $X=Y^{\mathbf{N}}$, where $Y \subseteq \mathbf{R}$ is non-empty. There is a social welfare function $W: X \rightarrow \mathbf{R}$ satisfying Lauwers' weak asymptotic Pareto LWAP and anonymity $A$ if and only if $Y$ is a bounded subset of $\mathbf{R}$.

\section{Part 2: The conflict between bounded anonymity and efficiency principles}

We study the compatibility of bounded anonymity with efficiency principles introduced in Sect. 3. Bounded anonymity is a stronger axiom than anonymity, and hence

\footnotetext{
${ }^{7}$ For all sets $A$ and $B$ let $A \backslash B$ denote the set $\{a \in A: a \notin B\}$.
} 
we expect bounded anonymity to put a harsher restriction on one period utility domains admitting a social welfare function. This is, however, not the case since Proposition 4.3 implies that there is a social welfare function satisfying BA and AP if $Y$ is finite. The channel in which BA limits the possibility of a welfare aggregator is another one: It turns out that BA limits the domains $Y$ admitting a binary welfare relation. We characterize one period utility domains admitting a binary welfare relation satisfying bounded anonymity and asymptotic Pareto. In Appendix B, we show that a similar characterization holds in the setting of Dubey and Mitra (2011), i.e., when weak Pareto WP is imposed. A quite remarkable consequence of the main result is that for a wide range of equity concepts $X$, the class of domains $Y$ with a binary welfare relation satisfying BA and $X$ coincides with the class of utility domains $Y$ admitting a social welfare function satisfying BA and $X$.

We summarize the main results of the second part of the paper in Theorem 5.1. The remainder of this section will be devoted to a proof of Theorem 5.1 and some implications/corollaries to the theorem.

Theorem 5.1 Let $X=Y^{\mathbf{N}}$, where $Y \subseteq \mathbf{R}$ is non-empty.

1. There is a binary welfare relation $\succsim_{R}$ on $X=Y^{\mathbf{N}}$, satisfying bounded anonymity $B A$ and approximate Pareto AP if and only if $Y$ is finite.

2. There is a binary welfare relation $\succsim_{R}$ on $X=Y^{\mathbf{N}}$, satisfying bounded anonymity $B A$ and weak approximate Pareto WAP if and only if $Y$ is bounded.

We only give a full proof of part 1 of theorem 5.1. The proof of part 2 uses similar arguments and is in Appendix A.5. The idea of proof is simple. We exhibit two sequences $x$ and $y$, such that $x$ can be obtained from $y$ using a bounded permutation, and such that $x$ Pareto dominates $y$ in the sense that a society of positive asymptotic density is strictly better off under $x$ compared to $y$. Bounded anonymity then implies that $x \sim y$, whereas asymptotic Pareto implies $x>y$. This yields the desired contradiction.

Proof One direction follows by part 2 of proposition 4.3. To show the other direction of the characterization result, let $X=Y^{\mathbf{N}}$ where $Y$ is some infinite subset of $\mathbf{R}$. Assume by way of contradiction that there is a binary welfare relation $\succsim^{\prime}$ on $X$ such that it satisfies BA and AP. Since $Y$ has total order $\geq$ induced from $\mathbf{R}$, by Lemma A. 3 there is a subset $Z \subseteq Y$ order isomorphic to $w$ or $w^{*}$. Assume that $Z$ is order isomorphic to $w$. Then there is an order isomorphism $f: \mathbf{N} \rightarrow Z$. Define a binary welfare relation $\succsim$ on $X=\mathbf{N}^{\mathbf{N}}$ by

$$
\begin{aligned}
& \left(x_{1}, \ldots, x_{n}, \ldots\right) \succsim\left(y_{1}, \ldots, y_{n}, \ldots\right) \\
& \Longleftrightarrow\left(f\left(x_{1}\right), \ldots, f\left(x_{n}\right), \ldots\right) \succsim^{\prime}\left(f\left(y_{1}\right), \ldots, f\left(y_{n}\right), \ldots\right)
\end{aligned}
$$

for all $x, y \in X$. Arguing as in the Proof of Proposition 4.4, part 1, it follows that $\succsim$ satisfies asymptotic Pareto AP and bounded anonymity BA. If $Z$ is order isomorphic to $w^{*}$, one can similarily show that this implies the existence of a binary welfare relation $\succsim$ on $X=\mathbf{N}^{\mathbf{N}}$ satisfying AP and BA. 
Hence, we have a binary welfare relation $\succsim$ on $X=\mathbf{N}^{\mathbf{N}}$ such that AP and BA holds. We show that this leads to a contradiction. Let $x$ and $y$ be the sequences

$$
\begin{aligned}
& x=(1,2,1,3,1,4,1,5,1, \ldots), \\
& y=(2,3,1,4,1,5,1,6,1, \ldots)
\end{aligned}
$$

with $x_{n}=n / 2+1$ if $n$ is even and $x_{n}=1$ otherwise; likewise, $y_{1}=2, y_{n}=2+n / 2$ if $n$ is even, and $y_{n}=1$ otherwise.

Let $\pi: \mathbf{N} \rightarrow \mathbf{N}$ be a permutation defined by $\pi(2):=1, \pi(1)=3, \pi(2 n):=2 n-2$ and $\pi(2 n-1):=2 n+1$ for all $n \geq 2$. It is then straightforward to verify that $\pi$ is a bounded permutation. Further $y_{\pi(1)}=y_{3}=1=x_{1}, y_{\pi(2)}=y_{1}=2=x_{2}$ and $y_{\pi(2 n)}=y_{2 n-2}=n+1=x_{2 n}$ and $y_{\pi(2 n-1)}=y_{2 n+1}=1=x_{2 n-1}$ for all $n \geq 2$ and hence it follows that $\pi \circ y=x$. By bounded anonymity BA of $\succsim$ we have $x=\pi \circ y \sim y$. Now notice that $y_{n} \geq x_{n}$ for all $n \in \mathbf{N}$, and moreover $y_{n}>x_{n}$ for all even numbers $n$. But since the lower asymptotic density of the set of even numbers is equal to $\frac{1}{2}$, hence positive, AP implies that $y \succ x$. Together with $x \sim y$ this yields a contradiction.

Remark: If a binary welfare relation $\succsim_{R}$ satisfies (bounded) anonymity, then $\succsim_{R}$ is reflexive. Hence, it is implicitly assumed that $\succsim_{R}$ is reflexive in Theorem 5.1.

The results in Sect. 4.2 imply that there is a social welfare function satisfying BA and AP if and only if $Y$ is finite. Thus we are naturally led to the question if there for every $Y \subseteq[0,1]$ is a social welfare order satisfying BA and AP. Theorem 5.1 shows that this is not the case. In fact, even if we lower our demands and hope for the possibility of a binary welfare relation $\succsim_{R}$ satisfying BA and AP, we hope for too much by Theorem 5.1 .

Theorem 4.1 (together with Proposition 4.3) implies that there is a social welfare function satisfying BA and AP (WAP) if and only if $Y$ is finite (bounded). The following is then an immediate corollary to Theorem 5.1.

Corollary 5.2 There is a binary welfare relation $\succsim$ on $X=Y^{\mathbf{N}}$ satisfying BA and AP (WAP) if and only if there is a social welfare function $W: X \rightarrow \mathbf{R}$ on $X$, satisfying $B A$ and $A P(W A P)$.

Theorem 5.1 limits the domains $Y$ admitting a binary welfare relation satisfying BA and AP (WAP). One may hope that relaxing BA by requiring A would make part of the negative result above go away. If we require a social welfare function satisfying A and AP, Theorem 4.1 implies that this is not the case. However, it may still be possible to find a binary welfare relation/order satisfying A and AP. Svensson (1980) shows, by use of the axiom of choice, that a social welfare order $\succsim o$ satisfying A and P exists on arbitrary domains $Y \subseteq[0,1]$. Hence, since $\mathrm{P} \Longrightarrow \mathrm{AP}$, there is a social welfare order satisfying A and AP whenever $Y \subseteq[0,1]$.

\section{Conclusion}

We introduced notions of efficiency and discussed their consistency with various equity axioms. More precisely we characterized one period utility domains admitting social 
welfare functions and/or relations satisfying efficiency and equity properties. Although perhaps mainly a technical endeavor, a discussion of welfare criteria is not really meaningful until the limitations imposed by them are known. First of all, it is important to know if there is any social welfare aggregator with the desired properties, since otherwise the properties are logically inconsistent. Once existence is established, it is nice to know whether it is possible to find such aggregators on arbitrary domains, or if not, what the exact limitations are. This perhaps has some technical value, but even normatively such a result could be useful, since it saves decision makers from engaging in a futile search for aggregation procedures that do not exist. A corollary to the results obtained in this paper is that there is a binary welfare relation satisfying bounded anonymity and (weak) asymptotic Pareto if and only if there is a social welfare function with such properties. It would be interesting to see if this result continues to hold for other equity notions as well. In Appendix, we verify that the result holds in the setting of Dubey and Mitra (2011), i.e., whenever the weak Pareto axiom is imposed.

Open Access This article is distributed under the terms of the Creative Commons Attribution 4.0 International License (http://creativecommons.org/licenses/by/4.0/), which permits unrestricted use, distribution, and reproduction in any medium, provided you give appropriate credit to the original author(s) and the source, provide a link to the Creative Commons license, and indicate if changes were made.

\section{A Appendix: Proofs}

\section{A .1 Proof of Lemma 4.2}

Proof For each positive integer $n$ :

$$
\begin{aligned}
& \mid\{k: k \leq n \text { and } n<\pi(k)\}|+|\{k: k \leq n \text { and } n \geq \pi(k)\} \mid=n, \\
& \mid\{k: k>n \text { and } n \geq \pi(k)\}|+|\{k: k \leq n \text { and } n \geq \pi(k)\} \mid=n .
\end{aligned}
$$

Consequently,

$$
\mid\{k: k \leq n \text { and } n<\pi(k)\}|=|\{k: k>n \text { and } n \geq \pi(k)\} \mid
$$

So $\lim _{n \rightarrow \infty} \frac{|\{k: k \leq n<\pi(k)\}|}{n}=0$ implies that $\lim _{n \rightarrow \infty} \frac{|\{k: \pi(k) \leq n<k\}|}{n}=0$. By boundedness of $Y$, there is an $M \in \mathbf{R}$ s.t $\left|x_{k}\right| \leq M$ for all $k$. Let $\varepsilon>0$. Since $\pi \in G$, there is a $N \in \mathbf{N}$ such that $\frac{|\{k: k \leq n<\pi(k)\}|}{n}<\frac{\varepsilon}{2 M}$ and $\frac{|\{k: \pi(k) \leq n<k\}|}{n}<\frac{\varepsilon}{2 M}$ for all $n \geq N$.

Further, note that the following equality of sets holds ${ }^{8}$

$$
\{k \leq n: k=\pi(l), l \leq n\}=\pi(\{k \leq n: \pi(k) \leq n\}) .
$$

Hence, since $\pi$ is a bijection, it follows that

\footnotetext{
8 With a slight abuse of notation we denote by $\{k \leq n: k=\pi(l), l \leq n\}$ the set $\{k \in \mathbf{N}: k \leq n, k=$ $\pi(l)$ for some $l \in \mathbf{N}$ with $l \leq n\}$. We use similar abbreviations throughout the proof.
} 


$$
\sum_{k \leq n: k=\pi(l), l \leq n} x_{k}=\sum_{k \leq n: \pi(k) \leq n} x_{\pi(k)} .
$$

Let $n \geq N$. Now rewrite the sum we wish to estimate as follows:

$$
\begin{aligned}
& \frac{1}{n} \sum_{k=1}^{n}\left(x_{k}-x_{\pi(k)}\right)=\frac{1}{n}\left(\sum_{k=1}^{n} x_{k}-\sum_{k=1}^{n} x_{\pi(k)}\right) \\
& =\frac{1}{n}\left(\sum_{k \leq n: k=\pi(l), l>n} x_{k}-\sum_{k \leq n: \pi(k)>n} x_{\pi(k)}\right) .
\end{aligned}
$$

We proceed to estimate the sum in (3) above.

$$
\begin{array}{r}
\left|\frac{1}{n} \sum_{k=1}^{n}\left(x_{k}-x_{\pi(k)}\right)\right|=\left|\frac{1}{n}\left(\sum_{k \leq n: k=\pi(l), l>n} x_{k}-\sum_{k \leq n: \pi(k)>n} x_{\pi(k)}\right)\right| \\
\leq \frac{M}{n}|\{k \leq n: k=\pi(l), l>n\}|+\frac{M}{n}|\{k: k \leq n<\pi(k)\}| \\
\leq \frac{M}{n}|\{k: \pi(k) \leq n<k\}|+\frac{M}{n}|\{k: k \leq n<\pi(k)\}| \leq \varepsilon .
\end{array}
$$

The first (in)equality follows by equation (2). The second (in)equality follows by the triangle inequality and the fact that $\left(x_{n}\right)$ is a sequence bounded by $M \in \mathbf{R}$. The third in(equality) follows since $\pi^{-1}(\{k \leq n: k=\pi(l), l>n\}) \subseteq\{k: \pi(k) \leq n<k\}$. By the estimates above we have $\left|W_{n}(x)-W_{n}(\pi \circ x)\right|<\varepsilon$ for all $n \geq N$, so $\lim _{n \rightarrow \infty}\left(W_{n}(x)-W_{n}(\pi \circ x)\right)=0$, as we had to show!

\section{A.2 Proof of Lemma 4.5}

In the Proof of Lemma 4.5 we will need the following well-known result.

Lemma A.1 The binary Van der Corput sequence $\left(d_{n}\right)$ is uniformly distributed in the unit interval $(0,1)$. The topological closure of $\left(d_{n}\right)$ is the closed unit interval $[0,1]$.

Proof The proof follows by theorem 1.1 (page 89) together with theorem 3.5 (page 127) in Kuipers and Niederreiter (2012).

We will also need the following intuitive result about asymptotic densities:

Lemma A.2 If $A \subseteq \mathbf{N}$ is such that $d(A)>0$, and $B$ is a finite subset of $A$, then $d(A \backslash B)>0$.

Proof Straightforward and hence omitted.

We now give our Proof of Lemma 4.5. 
Proof Assume $Y=\mathbf{N}$ and $X=Y^{\mathbf{N}}$ and that there is a social welfare function $W: X \rightarrow \mathbf{R}$ such that $W$ is asymptotically paretian AP and anonymous A. We shall prove that this leads to a contradiction. Let $\left(d_{n}\right)$ be the binary Van der Corput sequence, and for each real number $t \in(0,1)$ let $A(t):=\left\{n \in \mathbf{N}: d_{n} \in[0, t)\right\}$ and $B(t):=\left\{n \in \mathbf{N}: d_{n} \in[t, 1)\right\}$.

For each real $t \in(0,1)$ recursively define a subsequence of the natural numbers as follows. Set $n_{1}(t):=\min \{n \in \mathbf{N}: n \in A(t)\}$. Note that $n_{1}(t)$ is well defined, since $\mathbf{N}$ is well ordered and since the Van der Corput sequence is dense in $[0,1]$. Assume that $n_{1}(t), n_{2}(t), \ldots, n_{k-1}(t)$ has been defined such that $n_{1}(t)<n_{2}(t)<\cdots<n_{k-1}(t)$, and set $n_{k}(t):=\min \left\{n \in \mathbf{N}: n \in A(t) \backslash\left\{n_{1}(t), \ldots, n_{k-1}(t)\right\}\right\}$ (which by similar reasoning is well defined).

For every real number $t \in(0,1)$, define a sequence $x(t) \in X$ by:

$$
x_{n}(t)= \begin{cases}2 s+1 & \text { if } n=n_{s}(t) \text { forsome } s \in \mathbf{N} \\ 1 & \text { otherwise. }\end{cases}
$$

STEP 1: Let $\alpha, \beta \in(0,1)$ be such that $\beta>\alpha$ and let $N=\min \left\{n \in \mathbf{N}: d_{n} \in[\alpha, \beta)\right\}$. We show that $x_{n}(\beta) \geq x_{n}(\alpha)$ for all $n \in \mathbf{N}$ and $x_{n}(\beta)-x_{n}(\alpha) \geq 2$ for all $n \in A(\alpha)$ with $n>N$.

If $n=N$ then $x_{N}(\alpha)=1$ but $x_{N}(\beta)>1$. Let $n<N$ and $n \in A(\alpha)$. Then $n \in A(\beta)$ and since $N=\min \left\{n \in \mathbf{N}: d_{n} \in[\alpha, \beta)\right\}$ it follows that $x_{n}(\beta)=x_{n}(\alpha)$.

Assume $n>N$ and $n \in A(\alpha)$, then $n \in A(\beta)$. Since $n \in A(\alpha)$ there is a $k \in \mathbf{N}$ such that $n=n_{k}(\alpha)$. Hence, since $x_{N}(\alpha)=1$ but $x_{N}(\beta)>1$ we have that $n=n_{k+l}(\beta)$ for some $l>0$. Thus we have $x_{n}(\beta)-x_{n}(\alpha)=2(k+l)+1-(2 k+1)=2 l \geq 2$.

If $n \in B(\alpha)$ and $n \in A(\beta)$ then $x_{n}(\alpha)=1$ and $x_{n}(\beta)=2 s+1$ for some $s \in \mathbf{N}$, so $x_{n}(\beta) \geq x_{n}(\alpha)$. If $n \in B(\alpha)$ and $n \in B(\beta)$, then $x_{n}(\alpha)=x_{n}(\beta)=1$.

STEP 2: For each $t \in(0,1)$ define a sequence $z(t)$ by:

$$
z_{n}(t)= \begin{cases}x_{n}(t)+1 & \text { if } n \in A(t), \\ x_{n}(t) & \text { otherwise. }\end{cases}
$$

It is then evident that $W(z(\alpha))>W(x(\alpha))$, since the asymptotic density of $A(\alpha)$ is greater than zero.

STEP 3: Let $\alpha, \beta \in(0,1)$ with $\beta>\alpha$, we show that $W(x(\beta))>W(z(\alpha))$.

As before, let $N=\min \left\{n \in \mathbf{N}: d_{n} \in[\alpha, \beta)\right\}$. If $n=N$ then $x_{N}(\alpha)=1$ and $x_{N}(\beta)>1$ and since $N \notin A(\alpha)$ it follows that $z_{N}(\alpha)=x_{N}(\alpha)<x_{N}(\beta)$. If $n \in A(\alpha)$ and $n>N$, then by step 1 it follows that $x_{n}(\beta) \geq x_{n}(\alpha)+2=z_{n}(\alpha)+1>z_{n}(\alpha)$.

If $n \in B(\alpha)$ we have by step 1 and by definition of $z(\alpha)$ that $z_{n}(\alpha)=x_{n}(\alpha) \leq x_{n}(\beta)$.

Thus it remains to consider $n \in A(\alpha)$ with $n<N$. Note that there are finitely many elements $n \in \mathbf{N}$ with $n<N$ and $n \in A(\alpha)$ and hence we may w.l.o.g. enumerate them 
as $\left\{l_{1}, \ldots, l_{m}\right\}=\{n \in \mathbf{N}: n<N, n \in A(\alpha)\}$ with $l_{1}<l_{2}<\cdots<l_{m}$. Since $l_{i}<N$ and $l_{i} \in A(\alpha)$ we have $z_{l_{i}}(\alpha)=x_{l_{i}}(\alpha)+1=x_{l_{i}}(\beta)+1$ for all $i \in\{1, \ldots, m\}$.

Our aim is to permute the coordinates $l_{1}, \ldots, l_{m}$ of $z(\alpha)$ in such away that $x_{n}(\beta) \geq$ $z_{\pi(n)}(\alpha)$ for all $n \in \mathbf{N}$ for some finite permutation $\pi$, and then use Anonymity A to show that $x(\beta)$ dominates $z(\alpha)$.

Let $L(\alpha, \beta):=A(\beta) \cap B(\alpha)=\left\{n \in \mathbf{N}: d_{n} \in[\alpha, \beta)\right\}$. Note that we have $x_{n}(\beta) \geq$ $x_{n}(\alpha)+2$ for all $n \in L(\alpha, \beta)$. Since there are infinitely many elements in $L(\alpha, \beta)$, we may pick $K_{1}, \ldots, K_{m}$ in $L(\alpha, \beta)$ such that $K_{1}<\cdots<K_{m}$. Define a finite permutation $\pi: \mathbf{N} \rightarrow \mathbf{N}$ by $\pi\left(l_{i}\right)=: K_{i}$ and $\pi\left(K_{i}\right)=l_{i}$ for all $i \in\{1, \ldots, m\}$ and $\pi(k)=k$ for all $k \notin\left\{l_{1}, \ldots, l_{m}\right\} \cup\left\{K_{1}, \ldots, K_{m}\right\}$. Furthermore define $z^{\prime}(\alpha) \in X$ by $z_{n}^{\prime}(\alpha):=z_{\pi(n)}(\alpha)$ for all $n \in \mathbf{N}$.

We claim that $z^{\prime}(\alpha) \leq x(\beta)$. If $n=l_{i}$ for some $i \in\{1, \ldots, m\}$ then $z_{l_{i}}^{\prime}(\alpha)=$ $z_{K_{i}}(\alpha)=x_{K_{i}}(\alpha)=1<2 s+1=x_{l_{i}}(\beta)$. If $n=K_{i}$ for some $i \in\{1, \ldots, m\}$, then $z_{K_{i}}^{\prime}(\alpha)=z_{l_{i}}(\alpha)=x_{l_{i}}(\alpha)+1=x_{l_{i}}(\beta)+1<x_{K_{i}}(\beta)$. The last inequality follows by the fact that for all $m, k, l, n \in \mathbf{N}$ if $x_{k}(t)=2 m+1$ and $x_{n}(t)=2 l+1$ with $k>n$ then $n_{m}(t)=k>n=n_{l}(t)$, and since the sequence $\left(n_{k}(t)\right)$ is increasing in $k$, this in turn implies that $m>l$. Hence, $x_{k}(t)=2 m+1 \geq 2 l+3=x_{n}(t)+2$.

Finally note that $z_{n}^{\prime}(\alpha)=z_{n}(\alpha)$ for all $n \notin\left\{l_{1}, \ldots, l_{m}\right\} \cup\left\{K_{1}, \ldots, K_{m}\right\}$. Together with previous paragraphs (of step 3) this implies that $x_{n}(\beta) \geq z_{n}^{\prime}(\alpha)$ for all $n \in \mathbf{N}$ and $x_{n}(\beta)>z_{n}^{\prime}(\alpha)$ for all $n>N$ and $n \in A(\alpha)$. But since the asymptotic density of $A(\alpha)$ is $\alpha$ and $\{1, \ldots, N\}$ is a finite set, it follows by Lemma A.2 that $d(\{n \in \mathbf{N}: n>$ $N, n \in A(\alpha)\})=\alpha>0$ and hence by AP we have $W(x(\beta))>W\left(z^{\prime}(\alpha)\right)$. Anonymity A implies that $W\left(z^{\prime}(\alpha)\right)=W(z(\alpha))$ and hence $W(x(\beta))>W(z(\alpha))$, as we had to show.

STEP 4: For every $t \in(0,1)$ define an interval $[W(x(t)), W(z(t))]$. By step 2 we have $W(x(t))<W(z(t))$. Thus the interval $[W(x(t)), W(z(t))]$ is non-empty and hence by density of the rational numbers we may pick a rational number $q_{t} \in[W(x(t)), W(z(t))]$ for every $t \in(0,1)$. Define a function $f:(0,1) \rightarrow \mathbf{Q}$ by $f(t):=q_{t}$. We claim that $f$ is injective. Let $s>t$, by step 3 it follows that $W(x(s))>W(z(t))$ and hence $[W(x(t)), W(z(t))]$ and $[W(x(s)), W(z(s))]$ are disjoint, in turn implying that $q_{s} \neq q_{t}$. Thus $f$ is an injection from $(0,1)$ to $\mathbf{Q}$ contradicting the countability of $\mathbf{Q}$.

\section{A.3 Proof of Proposition 4.4 part 1}

We will need the following elementary result from order theory in our proof of Proposition 4.4 part 1 .

Lemma A.3 Every infinite set $Y$ with total order ${ }^{9} \succsim$ contains a subset order isomorphic to $w$ or $w^{*}$.

Proof If $Y$ contains a countable well ordered set $X$ then $X$ has a subset $X^{\prime} \subseteq X$ order isomorphic to $w$. To see this pick a minimal element $x_{1} \in X$. Assume that $x_{1}, \ldots, x_{n}$

\footnotetext{
${ }^{9}$ Relation $\succsim$ is a total order if it is antisymmetric, transitive and complete. Relation $\succsim$ is antisymmetric if $x \succsim y$ and $y \succsim x$ implies $x=y$.
} 
have been chosen such that for all $k, l \in\{1, \ldots, n\}: x_{k} \succ x_{l}$ if and only if $k>l$, and such that $x_{i+1}$ is minimal in $X \backslash\left\{x_{1}, \ldots, x_{i}\right\}$ for all $i \in\{1, \ldots, n-1\}$. Let $x_{n+1}$ be a minimal element in $X \backslash\left\{x_{1}, \ldots, x_{n}\right\}$. Then, since $x_{n+1} \in X$ and $x_{n+1} \in X \backslash\left\{x_{1}, \ldots, x_{i}\right\}$ for all $i \in\{1, \ldots, n-1\}$, it follows by the induction hypothesis that $x_{n+1} \succ x_{i}$ for all $i \in\{1, \ldots, n\}$. This defines a sequence $\left(x_{n}\right)$ recursively. Let $X^{\prime}=x(\mathbf{N})$. Then $X^{\prime}$ is a subset order isomorphic to $\mathbf{N}$.

If $Y$ contains no countable well ordered set, then there is a countable subset $Z \subseteq Y$ such that $Z$ contains no smallest element. We may then recursively construct a sequence $\left(x_{n}\right)$ in $Z$ such that $x_{1} \succ x_{2} \succ \ldots$. It then follows that $x(\mathbf{N})$ with order $\succsim$ is order isomorphic to $w^{*}$.

We now give our Proof of Proposition 4.4 part 1:

Proof Let $X=Y^{\mathbf{N}}$, where $Y$ is some infinite subset of $\mathbf{R}$. Assume there is a social welfare function $W^{\prime}: X \rightarrow \mathbf{R}$ that satisfies asymptotic Pareto AP and finite anonymity A. Since $Y$ is totally ordered by the natural order $\geq$ induced from $\mathbf{R}$, by Lemma A.3 there is a subset $Z \subseteq Y$ order isomorphic to $w$ or $w^{*}$. Assume $Z$ is order isomorphic to $w$. Then there is an order isomorphism $f: \mathbf{N} \rightarrow Z$. Define $W: X \rightarrow \mathbf{R}$, where $X=\mathbf{N}^{\mathbf{N}}$, by $W(x):=W^{\prime}\left(f\left(x_{1}\right), \ldots, f\left(x_{n}\right), \ldots\right)$. It remains to show that $W$ is asymptotically paretian AP and anonymous A, which by Lemma 4.5 yields a contradiction. That $W$ satisfies anonymity $\mathrm{A}$ is clear. To see that $W$ satisfies AP, let $x, y \in X$ be such that $x \geq y$ and $x_{i}-y_{i}>0$ for all $i \in A$ for some $A \subseteq \mathbf{N}$ with $\underline{d}(A)>0$. Then since $f: \mathbf{N} \rightarrow Z$ is an order isomorphism, we have $f\left(x_{i}\right)-$ $f\left(y_{i}\right)>0$ for all $i \in A$ and $f\left(x_{i}\right) \geq f\left(y_{i}\right)$ for all $i \in \mathbf{N}$. But $W^{\prime}$ satisfies AP and hence $W^{\prime}\left(f\left(x_{1}\right), \ldots, f\left(x_{n}\right), \ldots\right)>W^{\prime}\left(f\left(y_{1}\right), \ldots, f\left(y_{n}\right), \ldots\right)$. By definition of $W$ it follows that $W\left(x_{1}, \ldots, x_{n}, \ldots\right)>W\left(y_{1}, \ldots, y_{n}, \ldots\right)$.

If $Y$ contains a set $Z$ order isomorphic to $w^{*}$, then there is an order isomorphism $g: \mathbf{M} \rightarrow Z$, where $\mathbf{M}$ is the set of negative integers. Now, consider a social welfare function $W: \mathbf{N}^{\mathbf{N}} \rightarrow \mathbf{R}$ defined by $W\left(x_{1}, \ldots, x_{n}, \ldots\right):=$ $-W^{\prime}\left(g\left(-x_{1}\right), \ldots, g\left(-x_{n}\right), \ldots\right)$. That $W$ satisfies anonymity $\mathrm{A}$ is easy to see. We show AP: Let $x, y \in X$ be such that $x \geq y$ and $x_{i}-y_{i}>0$ for all $i \in A$ for some $A \subseteq \mathbf{N}$ with $\underline{d}(A)>0$. Then since $g: \mathbf{M} \rightarrow Z$ is an order isomorphism we have $g\left(-y_{i}\right)-g\left(-x_{i}\right)>0$ for all $i \in A$ and $g\left(-y_{i}\right) \geq g\left(-x_{i}\right)$ for all $i \in \mathbf{N}$. Since $W^{\prime}$ satisfies AP we have $W^{\prime}\left(g\left(-x_{1}\right), \ldots, g\left(-x_{n}\right), \ldots\right)<W^{\prime}\left(g\left(-y_{1}\right), \ldots, g\left(-y_{n}\right), \ldots\right)$. By definition of $W$ it then follows that $W\left(x_{1}, \ldots, x_{n}, \ldots\right)>W\left(y_{1}, \ldots, y_{n}, \ldots\right)$. Since $W$ satisfies A and AP, we have a contradiction by Lemma 4.5.

\section{A.4 Proof of Proposition 4.4 part 2}

The following characterization of unbounded subsets of $\mathbf{R}$ is used in our proof of proposition 4.4 part 2.

Lemma A.4 Let $\varepsilon>0$. Then $Y \subseteq \mathbf{R}$ is unbounded if and only if there is a subset $Z \subseteq Y$, order isomorphic to $w$ or $w^{*}$, such that the following (distance) condition holds:

$$
0<\varepsilon<\delta(Z):=\inf \{|x-y|: x \neq y \text { and } x, y \in Z\} .
$$

That is the distance between any pair of distinct points in $Z$ is larger than $\varepsilon>0$. 
Proof If $Y$ is not bounded, then either $Y$ lacks an upper bound, or otherwise a lower bound. Suppose that $Y$ has no upper bound. Pick some $x_{1} \in Y$, then there is an $x_{2} \in Y$ such that $x_{2}>x_{1}+\varepsilon$. Assume that $x_{1}, \ldots, x_{n}$ have been chosen such that $x_{i+1}-x_{i}>\varepsilon$. Since $Y$ has no upper bound there is a point $x_{n+1} \in Y$ such that $x_{n+1}>x_{n}+\varepsilon$. This defines a sequence $\left(x_{n}\right)$ recursively. If we let $Z=x(\mathbf{N})$ and give $Z$ the order induced from $\mathbf{R}$, then $Z$ is order isomorphic to $w$ and has the property that $\delta(Z)>\varepsilon$. If $Y$ is not bounded below a similar argument shows that there is a subset $Z$ order isomorphic to $w^{*}$ such that $\delta(Z)>\varepsilon$ in equation (4) holds.

We now give our proof of proposition 4.4 part 2.

Proof Let $X=Y^{\mathbf{N}}$, where $Y$ is an unbounded subset of $\mathbf{R}$. Assume there is a social welfare function $W^{\prime}: X \rightarrow \mathbf{R}$ satisfying WAP and A. By Lemma A.4, $Y$ contains a subset order isomorphic to $w$ or $w^{*}$ such that every pair of distinct points are at distance $\varepsilon>0$ apart.

If $Y$ contains a subset $Z$ order isomorphic to $w$, with $\delta(Z)>\varepsilon$, then there is an order isomorphism $f: \mathbf{N} \rightarrow Z$ with the property that $f(x)-f(y)>\varepsilon$ if and only if $x-y>$ 0 for all $x, y \in \mathbf{N}$. Define $W: X \rightarrow \mathbf{R}$, where $X=\mathbf{N}^{\mathbf{N}}$, by $W\left(x_{1}, \ldots, x_{n}, \ldots\right):=$ $W^{\prime}\left(f\left(x_{1}\right), \ldots, f\left(x_{n}\right), \ldots\right)$. It remains to show that $W$ is asymptotically paretian AP and anonymous $\mathrm{A}$, which by Lemma 4.5 yields a contradiction. It is clear that $W$ satisfies anonymity A. To see that $W$ satisfies AP, let $x, y \in X$ be such that $x \geq y$ and $x_{i}-y_{i}>0$ for all $i \in A$ for some $A \subseteq \mathbf{N}$ with $\underline{d}(A)>0$. Then since $f: \mathbf{N} \rightarrow Z$ is an order isomorphism, we have $f\left(x_{i}\right)-f\left(y_{i}\right)>\varepsilon$ for all $i \in A$ and $f\left(x_{i}\right) \geq$ $f\left(y_{i}\right)$ for all $i \in \mathbf{N}$. But $W^{\prime}$ satisfies WAP and hence $W^{\prime}\left(f\left(x_{1}\right), \ldots, f\left(x_{n}\right), \ldots\right)>$ $W^{\prime}\left(f\left(y_{1}\right), \ldots, f\left(y_{n}\right), \ldots\right)$. By definition of $W$ it follows that $W\left(x_{1}, \ldots, x_{n}, \ldots\right)>$ $W\left(y_{1}, \ldots, y_{n}, \ldots\right)$.

If $Y$ does not contain a subset $Z$ order isomorphic to $w$ with $\delta(Z)>\varepsilon$, then by Lemma A.4 $Y$ contains a subset $Z$ order isomorphic to $w^{*}$ with $\delta(Z)>\varepsilon$. In this case $W\left(x_{1}, \ldots, x_{n}, \ldots\right):=-W^{\prime}\left(g\left(-x_{1}\right), \ldots, g\left(-x_{n}\right), \ldots\right)$, where $g: \mathbf{M} \rightarrow Z$ is an order isomorphism, does the job. Note that there is an $\varepsilon>0$ such that $g(x)-g(y)>\varepsilon$ if and only if $x-y>0$ for all $x, y \in \mathbf{M}$. Anonymity A of $W$ is obvious. We show AP. Let $x, y \in X$ be such that $x \geq y$ and $x_{i}-y_{i}>0$ for all $i \in A$ for some $A \subseteq \mathbf{N}$ with $\underline{d}(A)>0$, then since $g: \mathbf{M} \rightarrow Z$ is an order isomorphism, we have $g\left(-y_{i}\right)-g\left(-x_{i}\right)>\varepsilon$ for all $i \in A$ and $g\left(-y_{i}\right) \geq g\left(-x_{i}\right)$ for all $i \in \mathbf{N}$. But $W^{\prime}$ satisfies WAP and hence $W^{\prime}\left(g\left(-x_{1}\right), \ldots, g\left(-x_{n}\right), \ldots\right)<W^{\prime}\left(g\left(-y_{1}\right), \ldots, g\left(-y_{n}\right), \ldots\right)$. By definition of $W$ it then follows that $W\left(x_{1}, \ldots, x_{n}, \ldots\right)>W\left(y_{1}, \ldots, y_{n}, \ldots\right)$.

\section{A.5 Proof of theorem 5.1 part 2}

Proof One direction follows by part (1) of proposition 4.3. To show the other direction of the characterization result, let $X=Y^{\mathbf{N}}$ where $Y$ is some unbounded subset of $\mathbf{R}$. Assume by way of contradiction that there is a binary welfare relation $\succsim^{\prime}$ on $X$ satisfying BA and WAP. Since $Y$ has a total order $\geq$ induced from $\mathbf{R}$, by Lemma A.4 there is a subset $Z \subseteq Y$ order isomorphic to $w$ or $w^{*}$ such that $0<\varepsilon<\delta(Z)=$ $\inf \{|x-y|: x \neq y$ and $x, y \in Z\}$. Assume that $Z$ is order isomorphic to $w$. Then there is an order isomorphism $f: \mathbf{N} \rightarrow Z$. Define a binary welfare relation $\succsim$ on 
$X=\mathbf{N}^{\mathbf{N}}$ by

$$
\begin{aligned}
& \left(x_{1}, \ldots, x_{n}, \ldots\right) \succsim\left(y_{1}, \ldots, y_{n}, \ldots\right) \\
& \quad \Longleftrightarrow\left(f\left(x_{1}\right), \ldots, f\left(x_{n}\right), \ldots\right) \succsim^{\prime}\left(f\left(y_{1}\right), \ldots, f\left(y_{n}\right), \ldots\right)
\end{aligned}
$$

for all $x, y \in X$. By arguing as in the Proof of Proposition 4.4, part 2, it follows that $\succsim$ satisfies asymptotic Pareto AP and bounded anonymity BA.

Hence, we have a binary welfare relation $\succsim$ on $X=\mathbf{N}^{\mathbf{N}}$ such that BA and AP holds. From here on the proof is exactly the same as the proof of part 1 of Theorem 5.1.

\section{B Appendix: Bounded anonymity and weak Pareto}

We now consider the consistency of bounded anonymity BA with weak Pareto WP. Dubey and Mitra (2011) obtain a characterization of domains admitting a social welfare function satisfying finite anonymity A and WP. The main result of this section shows that, by strengthening anonymity to bounded anonymity, the same domain restriction as in Dubey and Mitra (2011) gives a characterization of domains admitting a binary welfare relation satisfying A and WP. Existence in Dubey and Mitra (2011) is established by showing that the function $W_{\alpha}: X \rightarrow \mathbf{R}$ with $\alpha \in(0,1)$ defined by:

$$
W_{\alpha}(x):=\alpha \inf \left\{x_{n}: n \in \mathbf{N}\right\}+(1-\alpha) \sup \left\{x_{n}: n \in \mathbf{N}\right\}
$$

for all $x \in X$ satisfies WP and A. Since $W_{\alpha}$ clearly also satisfies BA their arguments imply the existence of a binary welfare relation (function) satisfying WP and BA whenever $Y$ contains no subset order isomorphic to $\mathbf{Z}$.

Proposition B. 1 Let $X=Y^{\mathbf{N}}$, then there is a binary welfare relation $\succsim$ on $X$ satisfying bounded anonymity BA and weak Pareto WP if and only if $Y$ contains no subset $Z$ of $\mathbf{R}$ order isomorphic to $\mathbf{Z}$.

Proof One direction follows by the discussion preceeding proposition B. 1 above. To show the other direction of the proposition, let $X=Y^{\mathbf{N}}$ and assume that $Y$ contains a subset $Z$ of $\mathbf{R}$ order isomorphic to $\mathbf{Z}$. Hence, there is an order isomorphism $f: \mathbf{Z} \rightarrow$ $Z$. Assume by way of contradiction that there is a binary welfare relation $\succsim^{\prime}$ on $X$ satisfying BA and WP. Define a binary welfare relation $\succsim$ on $X=\mathbf{Z}^{\mathbf{N}}$ by

$$
\begin{aligned}
& \left(x_{1}, \ldots, x_{n}, \ldots\right) \succsim\left(y_{1}, \ldots, y_{n}, \ldots\right) \\
& \quad \Longleftrightarrow\left(f\left(x_{1}\right), \ldots, f\left(x_{n}\right), \ldots\right) \succsim^{\prime}\left(f\left(y_{1}\right), \ldots, f\left(y_{n}\right), \ldots\right)
\end{aligned}
$$

for all $x, y \in X$. It is straightforward to check that $\succsim$ satisfies bounded anonymity BA. We show that $\succsim$ satisfies WP. Let $x, y \in X$ be such that $x_{i}>y_{i}$ for all $i \in \mathbf{N}$. Then $f\left(x_{i}\right)-f\left(y_{i}\right)>0$ for all $i \in \mathbf{N}$ implying that $\left(f\left(x_{1}\right), \ldots, f\left(x_{n}\right), \ldots\right) \succ^{\prime}$ $\left(f\left(y_{1}\right), \ldots, f\left(y_{n}\right), \ldots\right)$ and hence $\left(x_{1}, \ldots, x_{n}, \ldots\right) \succ\left(y_{1}, \ldots, y_{n}, \ldots\right)$. 
Hence, we have a binary welfare relation $\succsim$ on $X=\mathbf{Z}^{\mathbf{N}}$ such that BA and WP holds. We show that this leads to a contradiction. Let $x$ and $y$ be the sequences

$$
\begin{aligned}
& x=(-1,1,-2,2,-3,3,-4,4,-5, \ldots), \\
& y=(1,2,-1,3,-2,4,-3,5,-4, \ldots)
\end{aligned}
$$

with $x_{n}=n / 2$ if $n$ is even and $x_{n}=-(n-1) / 2-1$ otherwise; likewise, $y_{1}=1$, $y_{n}=1+n / 2$ if $n$ is even, and $y_{n}=-(n-1) / 2$ otherwise.

Let $\pi: \mathbf{N} \rightarrow \mathbf{N}$ be a permutation defined by $\pi(2):=1, \pi(1):=3$ and $\pi(2 n):=$ $2 n-2$ and $\pi(2 n-1)=2 n+1$ for all $n \geq 2$. It is easy to verify that $\pi$ is a bounded permutation and since $\pi \circ y=x$ it follows by bounded anonymity BA of $\succ$ that $x \sim y$. But notice that $y_{n}>x_{n}$ for all $n \in \mathbf{N}$. Hence, WP implies that $y \succ x$ which together with $x \sim y$ yields a contradiction.

\section{References}

Alcantud, J.C.R.: Inequality averse criteria for evaluating infinite utility streams: the impossibility of weak Pareto. J. Econ. Theory 147(1), 353-363 (2012)

Alcantud, J.C.R.: The impossibility of social evaluations of infinite streams with strict inequality aversion. Econ. Theory Bull. 1(2), 123-130 (2013)

Banach, S.: Theory of Linear Operations, vol. 38. Elsevier, Amsterdam (1987)

Banerjee, K.: On the equity-efficiency trade off in aggregating infinite utility streams. Econ. Lett. 93(1), 63-67 (2006)

Basu, K., Mitra, T.: Aggregating infinite utility streams with intergenerational equity: the impossibility of being paretian. Econometrica 71(5), 1557-1563 (2003)

Basu, K., Mitra, T.: Utilitarianism for infinite utility streams: a new welfare criterion and its axiomatic characterization. J. Econ. Theory 133(1), 350-373 (2007)

Basu, K., Mitra, T. (2007). Possibility theorems for equitably aggregating infinite utility streams. In: Intergenerational Equity and Sustainability, pp. 69-84. Springer, New York (2007)

Bossert, W., Sprumont, Y., Suzumura, K.: Ordering infinite utility streams. J. Econ. Theory 135(1), 579-589 (2007)

Crespo, J.A., Nuñez, C., Rincón-Zapatero, J.P.: On the impossibility of representing infinite utility streams. Econ. Theory 40(1), 47-56 (2009)

Debreu, G.: Representation of a preference ordering by a numerical function. Decis. Process. 3, 159-165 (1954)

Diamond, P.A.: The evaluation of infinite utility streams. Econometrica 33(1), 170-177 (1965)

Dubey, R.S., Mitra, T.: On equitable social welfare functions satisfying the weak Pareto axiom: a complete characterization. Int. J. Econ. Theory 7(3), 231-250 (2011)

Dubey, R.S., Mitra, T.: On social welfare functions on infinite utility streams satisfying Hammond Equity and Weak Pareto axioms: a complete characterization. Econ. Theory Bull. 3(2), 169-180 (2015)

Fey, M.: May's theorem with an infinite population. Soc. Choice Welf. 23(2), 275-293 (2004)

Fey, M.: An application of asymptotic density to characterizing voting rules. Tatra Mt. Math. Publ. 31(29), 29-37 (2005)

Fleurbaey, M., Michel, P.: Intertemporal equity and the extension of the Ramsey criterion. J. Math. Econ. 39(7), 777-802 (2003)

Hara, C., Shinotsuka, T., Suzumura, K., Xu, Y.: Continuity and egalitarianism in the evaluation of infinite utility streams. Soc. Choice Welf. 31(2), 179-191 (2008)

Koopmans, T.C.: Stationary ordinal utility and impatience. Econometrica 28(2), 287-309 (1960)

Kuipers, L., Niederreiter, H.: Uniform distribution of sequences. Courier Corporation (2012)

Lauwers, L.: Intertemporal objective functions: strong Pareto versus anonymity. Math. Soc. Sci. 35(1), 37-55 (1998) 
Lauwers, L.: Ordering infinite utility streams comes at the cost of a non-Ramsey set. J. Math. Econ. 46(1), 32-37 (2010)

Lauwers, L.: Strong anonymity and infinite streams. Discussion Paper 11.31, Center for Economic Studies, Katholieke Universiteit Leuven (2011)

Rudin, W.: Principles of Mathematical Analysis, vol. 3. McGraw-Hill, New York (1964)

Svensson, L.-G.: Equity among generations. Econometrica 48(5), 1251-1256 (1980)

Zame, W.R.: Can intergenerational equity be operationalized? Theor. Econ. 2(2), 187-202 (2007)

Zuber, S., Asheim, G.B.: Justifying social discounting: the rank-discounted utilitarian approach. J. Econ. Theory 147(4), 1572-1601 (2012) 